\title{
Effect of concurrent training on gender-specific biochemical variables and adiposity in obese adolescents
}

Barbara de Moura Mello Antunes 1,2, Diego Giuliano Destro Christofaro3, Paula Alves Monteiro ${ }^{1,2}$, Loreana Sanches Silveira' ${ }^{1,2}$, Rômulo Araújo Fernandes ${ }^{3}$, Jorge Mota ${ }^{4}$, Ismael Forte Freitas Júnior ${ }^{3}$

\begin{abstract}
Objective: The purpose of the present study was to analyze the effects of a 20-week concurrent training (20 WCT) intervention program on gender-specific body composition and metabolic variables in obese adolescents. Subjects and methods: Sample was composed of twenty-five obese adolescents, aged between 12 and $15(13.4 \pm 0.96)$ years. Fat-free mass (FFM), percentage trunk fat mass (TFM\%) and percentage fat mass (\%FM) were evaluated through dual-energy X-ray absorptiometry (DXA). Measurement of intra-abdominal adiposity (IAAT) was performed using ultrasound. Blood pressure was measured and blood samples analyzed for total cholesterol (TC), high-density lipoprotein cholesterol (HDL-c), low-density lipoprotein cholesterol (LDL-c), triglycerides (TG) and plasma glucose. All participants performed the concurrent training (combination of weight training and aerobic training) three times per week, one hour per day, for 20 weeks. Descriptive analysis and analysis of variance (ANOVA) for repeated measures were used to compare baseline, 10 week and 20 week moments using the Bonferroni post-hoc test. Statistical significance was set at $p<0.05$. Significant decrease inTC, LDL$c$ and TFM\% were verified in both genders after the 10 initial weeks of concurrent training. Results: $A$ significant increase in height was found in both the male and female groups $(p=0.001$ and $p=0.047$, respectively), after 20 weeks of concurrent training. In addition, several modifications were observed in body composition and metabolic variables, with a significant decrease in $\mathrm{BMI}(\mathrm{p}=0.002$ and $\mathrm{p}=$ $0.017)$, BMl z-score $(p=0.033$ and $p=0.004), F M \%(p=0.002$ and $p=0.002)$, TFM $\%(p=0.009$ and $p=$ $0.018)$, TC $(p=0.042$ and $p=0.001)$ and LDL-c $(p=0.006$ and $p=0.001)$ in the male and female groups, respectively, after 20 weeks of intervention when compared with baseline. Conclusion: Our results identified that concurrent training was an effective intervention for treating metabolic variable and body composition disorders, in both genders, by decreasing adiposity with consequent improvement in BMI and BMI z-scores, and enhancement in lipid profile variables. Arch Endocrinol Metab. 2015;59(4):303-9
\end{abstract}

Keywords

Training; obesity; adolescent; body composition; lipid profile
1 Instituto de Biociências, Universidade Estadual Paulista (Unesp), Rio Claro, SP, Brazil 2 Grupo de Pesquisa, Exercício e Imunometabolismo, Departamento de Educação Física, Unesp, Presidente Prudente, SP, Brazil ${ }^{3}$ Departamento de Educação Física, Unesp, Presidente Prudente, SP, Brazil

${ }^{4}$ Centro de Pesquisa em Atividade Física, Saúde e Lazer, Faculdade de Desporto da Universidade do Porto, Porto, Portugal

Correspondence to:

Barbara de Moura Mello Antunes Departamento de Educação Física, Universidade Estadual Paulista Rua Roberto Simonsen, 305, 19060-900 - Presidente Prudente SP, Brazil

bah_tunes@hotmail.com

Received on Sept/26/2014 Accepted on May/18/2015

DOI: 10.1590/2359-3997000000095

\section{INTRODUCTION}

$\mathrm{E}$ pidemiological evidence indicates obesity as a relevant concern in the pediatric population $(1,2)$. It is well documented that obesity constitutes a risk factor for other chronic outcomes, such as insulin resistance (3), elevated blood pressure (4), dyslipidemia (5) and nonalcoholic fatty liver disease (6). Moreover, obesity triggered in childhood tends to continue into adulthood (7).

The specificity of the distribution and accumulation of adiposity varies according to gender. Females present a higher concentration of adipose tissue in the subcutaneous gluteofemoral region and males a higher concentration of fat mass in the abdominal region. These differences are due to the influence of hormones such as estrogen, testosterone, cortisol and growth hormone (8).
Independent of age, physical exercise and dietary control are widely prescribed to treat obesity $(9,10)$. In addition, there are a wide variety of exercise protocols targeting weight loss, although their effectiveness is not clear when applied to pediatric population. Aerobic and strength exercises have been used to reduce fat mass and improve fasting glucose and lipid metabolism $(11,12)$ however, despite the effect of both methods on weight loss programs, there is not sufficient evidence that strength training (ST) promotes significant improvements in the metabolic profile $(13,14)$. Furthermore, it is not clear if concurrent training increases fat-free mass and/or decreases fat mass and whether it improves metabolic parameters in obese adolescents. 
Thus, the purpose of the present study was to analyze the effects of a 20 -week concurrent training (20 WCT) intervention program on body composition and metabolic variables, according to gender, in obese adolescents.

\section{SUBJECTS AND METHODS}

\section{Subjects}

The subjects were invited through television and newspaper advertising to participate in a weight loss intervention program for obese adolescents, conducted at the Universidade Estadual Paulista (Unesp) in Presidente Prudente, Brazil. The participants contacted the researchers by telephone and an appointment was scheduled to take measurements at the Universidade Estadual Paulista - Unesp in the Study Center and Laboratory of Assessment and Prescription of motor activity (Celapam).

The preliminary diagnosis of obesity was performed using body mass index (BMI) (15). After this preliminary diagnosis, the following inclusion criteria were used to select the subjects: i) aged 12 to 15 years; ii) no engagement in regular physical activity within the three months immediately prior to the study; iii) no restrictions on physical activity diagnosed by a medical doctor; iv) a consent form signed by the parents/guardians to participate in the study. One hundred and eighty obese children and adolescents (97 male and 83 female [aged 7-16 years]) contacted the researchers and the exercise protocol began with 80 subjects who met all the inclusion criteria. After 10 weeks of study, the sample had a loss of $50 \%$ subjects $(n=40)$ due to intervention time and duration, number of missed sessions, health problems and/or termination without justification. Finally, only 25 subjects ( 13 male and 12 female) concluded the 20 weeks exercise and evaluation protocol.

The present study was approved by the Ethical Research Committee of the Universidade Estadual Paulista - Campus of Presidente Prudente (Unesp) (protocol number $07 / 2009)$.

\section{Body composition}

\section{Dual-energy x-ray absorptiometry (DEXA)}

After the preliminary diagnosis of obesity through BMI, body composition (whole-body and segmental) was estimated using dual-energy X-ray absorptiometry (DEXA) (Lunar DPX-NT scanner [Lunar DPX-NT;
General Electric Healthcare, Little Chalfont, Buckinghamshire, United Kingdom] software version 4.7). Fatfree mass (FFM), percentage trunk fat mass (TFM\%) and percentage fat mass $(\% \mathrm{FM})$ were estimated. All measurements were carried out at the laboratory of the university in a climate-controlled room and the equipment was calibrated every morning, as recommended by the manufacturer.

\section{Intra-abdominal adipose tissue}

An ultrasound examination of the upper abdomen was performed to measure intra-abdominal adiposity (IAAT). The examination was performed by only one qualified radiologist, using a TOSHIBA Eccocee with a 3.7 Mhz convex transducer. All adolescents were instructed to fast for 4 hours prior to the evaluation, according to medical literature.

\section{Blood samples}

Blood samples were collected from subjects in tubes containing EDTA after a 12 hours fasting period. All blood sample collections (performed by nurses) and biochemical analyses were carried out in a private laboratory. Fasting blood measurements were performed using the colorimetric method. Total cholesterol (TC), high-density lipoprotein cholesterol (HDL-c), lowdensity lipoprotein cholesterol (LDL-c), triglycerides and plasma glucose were analyzed (16).

\section{Blood pressure}

Measurements of systolic blood pressure (SBP) and diastolic blood pressure (DBP) were performed using automatic equipment (Omron HEM 742) on the right arm with the subject lying down, resting and after a 15 minutes rest period.

\section{Training program}

The exercise protocol was based on 30-minute sessions of aerobic activities (walking and jogging) followed by 30 minutes of strength exercises (resistance training), three times per week, over a period of 20 weeks (17).

The 10-repetition maximum (10RM) protocol was used to predict the maximum amount of resistance that could be actively overcome in 1 repetition. The strength exercises were: 45-degree leg press, seated row, chest press, squat, upper chest, leg workout, bicep curl, pec deck, triceps extension, quadriceps extension, sit ups and dorsal raise. 
The series of strength exercises consisted of four weeks of neuromuscular adaptation, followed by 16 weeks of training, as follows:

$\mathrm{I}^{\text {st }}-4^{\text {th }}$ weeks $\rightarrow$ Neuromuscular adaptation with light loads: strength exercises (single set of 20 repetitions) + sit ups (single set of 15 repetitions) + dorsal raise (single set of 15 repetitions).

$5^{\text {th }}$ to $8^{\text {th }}$ weeks $\rightarrow$ Exercise at $40 \%$ of $10 \mathrm{RM}$ : strength exercises (single set of 20 repetitions) + sit ups (single set of 20 repetitions) + dorsal raise (single set of 20 repetitions).

$9^{\text {th }}$ to $12^{\text {th }}$ weeks $\rightarrow$ Exercises at $45 \%$ 10RM: strength exercises (single set of 20 repetitions) + sit ups (single set of 20 repetitions) + dorsal raise (single set of 20 repetitions).

$13^{\text {th }}$ to $16^{\text {th }}$ weeks $\rightarrow$ Exercises at $50 \% 10 \mathrm{RM}:$ strength exercises (single set of 20 repetitions) + sit ups (single set of 20 repetitions) + dorsal raise (single set with 20 repetitions).

$17^{\text {th }}$ to $20^{\text {th }}$ weeks $\rightarrow$ Exercises at $55 \%$ 10RM: strength exercises (single set of 20 repetitions) + sit ups (single set of 20 repetitions) + dorsal raise (single set of 20 repetitions).

The periodization for aerobic exercise was composed of four weeks of adaptation, concomitant with strength exercises in the same session, which consisted of low/moderate-intensity walking for 30 minutes without heart rate control. After these four weeks, the aerobic activities (walking and jogging) were adjusted according to the maximum heart rate ( $65 \%$ to $85 \%)$ values provided by the maximal oxygen consumption test. The aerobic activities were performed on a running track with each workload specific to each individual.

The intensity of aerobic exercise was monitored using a heart hate monitor (Polar ${ }^{\circledR}$ S810), placed, randomly, on four individuals per session. The participants were advised to drink water and wear appropriate exercise clothing.

\section{Statistical analysis}

Data normality was verified using the KolmogorovSmirnov test. Numerical variables were presented as mean, standard-deviation and delta percentage $(\Delta \%)$ of the difference between time points. The sphericity of the data was verified by the Mauchly's test. When the sphericity was not found the Greenhouse-Geisser correction was used. An analysis of variance (ANOVA) for repeated measures was used to compare the three time points (baseline, 10 weeks and 20 weeks). To check for possible differences between time points, the Bonferroni post-hoc test was used. Statistical significance was set at $\mathrm{p}$-value $<5 \%$ and the statistical software SPSS (15.0) performed all analyses.

\section{RESULTS}

The descriptions of the sample for metabolic variables and body composition are shown in table 1 for males and table 2 for females and it can be seen that the sam-

Table 1. Effects of concurrent training according time points in male gender. Presidente Prudente, SP, Brazil

\begin{tabular}{lcccc}
\hline & \multicolumn{3}{c}{ Boys (n= 13) } \\
\cline { 2 - 5 } & Baseline & $\mathbf{1 0 W}$ & $\mathbf{2 0 W}$ & $\mathbf{p}$ \\
\hline Weight (kg) & $86.6 \pm 20.3$ & $86.5 \pm 20.7$ & $85.0 \pm 11.3$ & 0.264 \\
Height (cm) & $163.9 \pm 6.0$ & $164.6 \pm 5.6^{\mathrm{a}}$ & $165.5 \pm 5.5^{\mathrm{a}, \mathrm{b}}$ & $\mathbf{0 . 0 0 1}$ \\
BMl (kg/cm²) & $32.0 \pm 5.9$ & $31.7 \pm 6.0$ & $30.8 \pm 11.2^{\mathrm{a}}$ & $\mathbf{0 . 0 0 2}$ \\
BMI-z score & $2.04 \pm 0.4$ & $1.96 \pm 0.4^{\mathrm{a}}$ & $1.83 \pm 0.5^{\mathrm{a}, \mathrm{b}}$ & $\mathbf{0 . 0 3 3}$ \\
Glucose (mg/dL) & $87.0 \pm 4.8$ & $87.1 \pm 6.3$ & $87.0 \pm 4.4$ & 1.000 \\
TG (mg/dL) & $161.1 \pm 71.2$ & $128.3 \pm 55.3^{\mathrm{a}}$ & $143.8 \pm 54.4$ & $\mathbf{0 . 0 0 1}$ \\
TC (mg/dL) & $176.7 \pm 37.6$ & $163.1 \pm 32.8^{\mathrm{a}}$ & $163.9 \pm 33.1^{\mathrm{a}}$ & $\mathbf{0 . 0 4 2}$ \\
HDL-c (mg/dL) & $39.1 \pm 7.5$ & $40.5 \pm 7.9$ & $40.7 \pm 8.3$ & 0.958 \\
LDL-c (mg/dL) & $105.7 \pm 34.3$ & $93.7 \pm 32.5^{\mathrm{a}}$ & $\mathbf{0 . 0 0 6}$ \\
SBP & $129.5 \pm 12.2$ & $96.7 \pm 31.5^{\mathrm{a}}$ & $128.4 \pm 10.3$ & 0.870 \\
DBP & $73.3 \pm 6.5$ & $132.0 \pm 14.5$ & $70.8 \pm 6.0^{\mathrm{a}}$ & 0.068 \\
FM (\%) & $45.1 \pm 5.0$ & $71.8 \pm 7.7$ & $41.8 \pm 4.8^{\mathrm{a}}$ & $\mathbf{0 . 0 0 2}$ \\
FFM (kg) & $42.1 \pm 7.2$ & $42.7 \pm 5.3^{\mathrm{a}}$ & $44.5 \pm 6.5^{\mathrm{a}}$ & $\mathbf{0 . 0 0 5}$ \\
TFM (\%) & $47.4 \pm 3.8$ & $43.0 \pm 6.5$ & $43.9 \pm 3.9^{\mathrm{a}}$ & $\mathbf{0 . 0 0 9}$ \\
\hline
\end{tabular}

TG: triglycerides; TC: total cholesterol; HDL-c: high density lipoprotein cholesterol; LDL-C: low density lipoprotein cholesterol; SBP: systolic blood pressure; DBP: diastolic blood pressure; FM: fat mass percentage; FFM: fat-free mass; TFM: trunk fat mass. a: vs. baseline; ${ }^{\text {b: }}$ vs. 10 weeks. 
ple was composed of obese individuals by the combined analysis of all anthropometric variables. Parametric samples were presented as mean and standard deviation (mean $\pm \mathrm{SD}$ ) and non-parametric samples as median and interquartile range, according to gender, at baseline, after 10 weeks and after 20 weeks of intervention.

Table 1 demonstrates, through mean and standard deviation, alterations between baseline, the 10 initial weeks and the 10 final weeks in the male group. Reductions in BMI $z$-score $(\Delta \%=-3.9 ; \mathrm{p}=0.033), \mathrm{FM} \%$ $(\Delta \%=-5.3 ; \mathrm{p}=0.002), \mathrm{TFM} \%(\Delta \%=-5.1 ; \mathrm{p}=0.009)$, $\mathrm{TC}(\Delta \%=-7.7 ; \mathrm{p}=0.042)$ and LDL-c $(\Delta \%=-8.5 ; \mathrm{p}$ $=0.006) ;$ and an increase in height $(\Delta \%=+0.4 ; \mathrm{p}=$ $0.001)$, were observed after 10 weeks of intervention. In addition, reductions in $\mathrm{BMI}(\Delta \%=-3.8 ; \mathrm{p}=0.002)$, BMI z-score $(\Delta \%=-10.3)$, TC $(\Delta \%=-7.2)$, LDL-c $(\Delta \%$ $=-11.4), \mathrm{FM} \%(\Delta \%=-7.3)$ and $\mathrm{TFM} \%(\Delta \%=-7.4)$ were observed after 20 weeks of concurrent training when compared with baseline. TG concentration was the only variable to present a decrease $(\Delta \%=-20.4 ; \mathrm{p}=$ $0.001)$ after 10 weeks of training, although in the final 10 weeks of the concurrent training protocol a slight, not significant, increase was observed.

In the female group, decreases in TFM $\%(\Delta \%=-5.8$; $\mathrm{p}=0.018)$, TC $(\Delta \%=-17.0 ; \mathrm{p}=0.001)$ and LDL-c $(\Delta \%=-22.9 ; \mathrm{p}=0.001)$ were observed after 10 initial weeks of the intervention, however weight $(\Delta \%=-3.4$; $\mathrm{p}=0.047), \mathrm{BMI}(\Delta \%=-4.4 ; \mathrm{p}=0.017)$, BMI $\mathrm{z}$-score
$(\Delta \%=-5.1 ; \mathrm{p}=0.004), \mathrm{FM} \%(\Delta \%=-6.8 ; \mathrm{p}=0.002)$, $\mathrm{TFM} \%(\Delta \%=-7.5)$, TC $(\Delta \%=-15.9)$ and LDL-c $(\Delta \%$ $=-22.1)$ presented significant improvements, by reducing, after 20 weeks of concurrent training. In addition, a significant reduction was verified in TG concentration $(\Delta \%=-24.4 ; \mathrm{p}=0.003)$ after 10 weeks of training with a subsequent increase in the final weeks, although not significant. The description of the female group is shown in table 2 .

\section{DISCUSSION}

From the results of the present study it was possible to verify that concurrent training promoted significant beneficial alterations in body composition and metabolic variables in both genders. Significant improvements were also observed in lipid profile variables, in which the female gender demonstrated greater reductions. Recently, the American College of Sports Medicine (14) stated that there is a lack of evidence to indicate that strength training, without aerobic exercise or dietary control, is sufficient to promote weight loss and, therefore, its utilization in combination with aerobic activities is recommended. According to studies carried out with young girls, the combination of aerobic and strength exercises is more effective at decreasing body adiposity than either nutrition or one of these models of exercise in isolation (18).

Table 2. Effects of concurrent training according time points in female gender. Presidente Prudente, SP, Brazil

\begin{tabular}{lcccc}
\hline & & Girls $(\mathbf{n}=\mathbf{1 2})$ & $\mathbf{2 0 W}$ & $\mathbf{p}$ \\
\cline { 2 - 5 } & Baseline & $\mathbf{1 0 W}$ & $73.1 \pm 9.5^{\mathrm{a}}$ & $\mathbf{0 . 0 4 7}$ \\
\hline Weight (kg) & $75.7 \pm 10.5$ & $75.9 \pm 9.6$ & $161.4 \pm 5.2^{\mathrm{b}}$ & $\mathbf{0 . 0 4 7}$ \\
Height (cm) & $160.5 \pm 4.9$ & $161.2 \pm 5.2$ & $28.1 \pm 3.5^{\mathrm{a}}$ & $\mathbf{0 . 0 1 7}$ \\
BMl (kg/cm²) & $29.4 \pm 3.8$ & $29.2 \pm 3.5$ & $1.87 \pm 0.4^{\mathrm{a}}$ & $\mathbf{0 . 0 0 4}$ \\
BMI-z score & $1.97 \pm 0.4$ & $1.98 \pm 0.3$ & $80.5 \pm 4.3$ & 0.301 \\
Glucose (mg/dL) & $77.7 \pm 6.7$ & $80.6 \pm 4.6$ & $93.6 \pm 53.5$ & $\mathbf{0 . 0 0 3}$ \\
TG (mg/dL) & $102.5 \pm 44.0$ & $77.5 \pm 31.2^{\mathrm{a}}$ & $148.1 \pm 19.8^{\mathrm{a}}$ & $\mathbf{0 . 0 0 1}$ \\
TC (mg/dL) & $176.2 \pm 24.2$ & $146.2 \pm 20.1^{\mathrm{a}}$ & $45.7 \pm 10.1$ & 0.510 \\
HDL-c (mg/dL) & $48.9 \pm 11.4$ & $48.5 \pm 9.5$ & $83.0 \pm 20.7^{\mathrm{a}}$ & $\mathbf{0 . 0 0 1}$ \\
LDL-c (mg/dL) & $106.6 \pm 25.7$ & $82.2 \pm 22.4^{\mathrm{a}}$ & $121.0 \pm 11.4$ & 1.000 \\
SBP & $122.4 \pm 20.0$ & $122.8 \pm 14.4$ & $71.3 \pm 6.0$ & 1.000 \\
DBP & $72.0 \pm 8.5$ & $72.6 \pm 7.7$ & $42.6 \pm 4.9^{\mathrm{a}}$ & $\mathbf{0 . 0 0 2}$ \\
FM (\%) & $45.7 \pm 4.3$ & $44.2 \pm 5.9$ & $38.3 \pm 3.2$ & 0.230 \\
FFM (kg) & $37.4 \pm 3.2$ & $37.6 \pm 3.1$ & $44.3 \pm 5.8^{\mathrm{a}}$ & \\
TFM (\%) & $47.9 \pm 4.4$ & $45.1 \pm 8.0^{\mathrm{a}}$ & $\mathbf{0 . 0 1 8}$ \\
\hline
\end{tabular}

TG: triglycerides; TC: total cholesterol; HDL-c: high density lipoprotein cholesterol; LDL-c: low density lipoprotein cholesterol; SBP: systolic blood pressure; DBP: diastolic blood pressure; FM: fat mass percentage; FFM: fat-free mass; TFM: trunk fat mass. a: vs. baseline; b: vs. 10 weeks. 
Physical training is able to promote weight loss, as well as decreasing fat mass and increasing fat-free mass relative to the intensity of exercise (19). Losing weight and promoting healthy slimming requires moderate-intense exercise for about 60 minutes (14). LeMura and Maziekas (20) demonstrated that superior alterations in body composition of obese children and adolescents occurred as a result of concurrent training (aerobic and strength exercises) when performed at low-intensity over a long-duration.

The training protocol in the present study was considered as low-moderate intensity and significant improvements were observed in variables related to body composition, with significant decreases in percentage of adipose tissue and trunk fat mass, in both genders. However, the gain or reduction in these variables occurred at different time points in the intervention. In addition, the greatest reduction was observed when comparing the results after 20 weeks of intervention (total time) with the baseline in both groups.

In a study performed by Davis and cols. (18) with girls $(15.2 \pm 1.1$ years $)$ submitted to a pilot protocol of nutritional intervention and combined exercise (aerobic and strength), the authors observed that after 16 weeks of training there was a significant reduction of $3 \%$ in body adiposity corroborating with the present study that found a reduction of $3.4 \%$ after 20 weeks. According to research with boys, a combination of circuit-based resistance training and aerobic exercises over 12 weeks was responsible for a decrease of $2.2 \%$ in the percentage of body fat (21), however the results of the present study showed a reduction after the tenth week of intervention. Response to training in relation to total fat occurs differently between the sexes due to maturational processes. In both genders, there is an increase in body weight in the final stages of maturation. In girls there is a proportional increase between fat mass and fat-free mass, while males demonstrate an increase in fat-free mass and a decrease in fat mass (22).

After the 20 weeks training protocol, significant reductions in TFM\% were observed in both the male and female groups. Studies that compare the effects of aerobic and anaerobic training in obese adolescents verified a decrease of $6.5 \%$ and $8.2 \%$ respectively $(23)$. Several studies indicate that distribution of body fat may be an indicator of risk of cardiovascular diseases, alterations in blood pressure, cholesterol concentrations and glucose tolerance (24-26), whereas individuals with higher fat deposits in the upper body are more likely to present an increased risk of cardiovascular diseases. However, exercise may be a strong tool for reducing disease-related accumulations of fat in the trunk region.

Regarding lipid profile, TC, LDL-c and TG concentrations were positively affected by concurrent training, with significant decreases in all these metabolic parameters, except HDL-c, in both genders. The improvements in lipid profiles are directly related to the reduction in body mass and fat mass in the central regions of the body owing to decreases in free fatty acids and the formation of different lipoprotein molecules $(27,28)$. In agreement with our findings for LDL-c and TC, Foschini and cols. (29) found similar results for concurrent training after 14 weeks in obese youth. In the present study, the applied exercise protocol was composed of activities of low (in the initial protocol) and moderate intensity. Kraus and Slentz (30) reviewed the scientific literature and identified that, although lipids are the principle fuel for sustaining activities of moderate intensity, activities of low intensity have a relevant burden in the control of fasting serum lipids and should be encouraged in sedentary obese adolescents.

The present study suggested that intensity of effort may have influenced the positive effect of concurrent training, where lipid variables showed significant improvements after 20 weeks of intervention, except for HDL-c. In agreement with the literature it is believed that this result was due to the intensity progression during training, where intensity remained low to moderate in the first 10 weeks of training, as it was possible to observe decreased TG (baseline: 102.5; post: 77.5), TC (baseline: 176.2; post: 146.2) and LDL-c (baseline: 106.6; post: 82.2). However, when compared with the results of the first 10 weeks (Baseline-TG: 77.5, PostTG: 93.6; Baseline-TC: 146.2, Post-TC: 148.1; Baseline LDL-c: 82.2 , Post LDL-c: 83.0 ), there was no improvement.

When comparing results between genders, it was observed that both groups responded to training in the same variables, with significant alterations observed at the end of the intervention. A small difference was identified between the groups; male adolescents seemed to respond to training more quickly with a higher number of modifications after 10 weeks of the protocol. In a previous study by our group which analyzed the effects of concurrent training on risk factors and steatosis, significant alterations were identified in height, percentage body fat, fat mass, fat free mass, percentage trunk fat, total cholesterol and LDL-c (31), after 20 weeks. These 
findings suggest that this population requires a longer intervention time, given the limitations and biological stage, for significant changes to occur in body composition and lipid variables. In contrast, in the present study, it was demonstrated that after 10 weeks of intervention there were already significant enhancements in body composition and metabolic variables, regardless of gender, suggesting that a shorter intervention time, when controlled, could be an effective strategy. However, comparisons between genders were not performed and this is suggested for future research projects.

The limitations of the present study should be highlighted. The absence of a sedentary control group, according to gender, in addition to the fact that dietary habits were not recorded, are potential confounders of the results obtained. However, this study considered the biological individuality of the participants, prioritizing physiological, morphological and maturational features, according to gender, and comparing individuals at different moments of the intervention period. In addition the absence a maximal oxygen uptake test in the intermediate moment ( 10 weeks) and other metabolic profiles, such as insulin, were a limitation to better interpretation of the results.

In summary, our results identified that concurrent training seems to be an effective exercise protocol, able to promote significant improvements in body composition and metabolic variables, by decreasing fat free mass and trunk fat mass, and consequently BMI and BMI z-scores, and enhancing lipid profile variables, by reducing triglycerides, total cholesterol and LDL-c, for both genders.

Acknowledgments: Study Center and Laboratory of Assessment and Prescription of motor activity (Celapam).

Funding received: Coordenação de Aperfeiçoamento de Pessoal de Nivel Superior (Capes).

Disclosure: no potential conflict of interest relevant to this article was reported.

\section{REFERENCES}

1. Labree LJ, van de Mheen H, Rutten FF, Foets M. Differences in overweight and obesity among children from migrant and native origin: a systematic review of the European literature. Obes Rev. 2011;12(5):e535-47.

2. Corvalán C, Uauy R, Kain J, Martorell R. Obesity indicators and cardiometabolic status in 4-y-old children. Am J Clin Nutr. 2010;91(1):166-74.

3. Kalupahana NS, Moustaid-Moussa N, Claycombe KJ. Immunity as a link between obesity and insulin resistance. Mol Aspects Med. 2012;33(1):26-34.
4. Christofaro DG, Ritti-Dias RM, Fernandes RA, Polito MD, Andrade SM, Cardoso JR, et al. High blood pressure detection in adolescents by clustering overall and abdominal adiposity markers. Arq Bras Cardiol. 2011;96(6):465-70.

5. Klop B, Elte JW, Cabezas MC. Dyslipidemia in obesity: mechanisms and potential targets. Nutrients. 2013;5(4):1218-40.

6. Silveira LS, Monteiro PA, Antunes BMM, Seraphim PA, Fernandes RA, Christofaro DGD, et al. Intra-abdominal fat is related to metabolic syndrome and non-alcoholic fat liver disease in obese youth. BMC Pediatrics. 2013;13:115.

7. Hills AP, Andersen LB, Byrne NM. Physical activity and obesity in children. Br J Sports Med. 2011;45(11):866-70.

8. McArdle WD, Katch FI, Katch VL. Essentials of exercise physiology. Philadelphia: Lea and Febiger; 1994.

9. Van Gaal LF, Mertens IL, De Block CE. Mechanisms linking obesity with cardiovascular disease. Nature. 2006;444(7121):875-80.

10. Fernandes RA, Zanesco A. Early physical activity promotes lower prevalence of chronic diseases in adulthood. Hypertens Res. 2010;33(9):926-31.

11. Fernandes RA, Christofaro DG, Casonatto J, Codogno JS, Rodrigues EQ, Cardoso M, et al. A. Prevalence of dyslipidemia in individuals physically active during childhood, adolescence and adult age. Arq Bras Cardiol. 2011; 97(4):317-23.

12. Leite N, Lazarotto L, Cavazza JF, Lopes MFA, Bento PCB, Torres $R$, et al. Efeitos de exercícios aquáticos e orientação nutricional na composição corporal de crianças e adolescentes obesos. Rev Bras Cineantropom Desempenho Hum. 2010;12(4):232-8.

13. Braith RW, Stewart KJ. Resistance exercise training: its role in the prevention of cardiovascular disease. Circulation. 2006;113(22):2642-50.

14. Donnelly JE, Blair SN, Jakicic JM, Manore MM, Rankin JW, Smith BK; American College of Sports Medicine. American College of Sports Medicine Position Stand. Appropriate physical activity intervention strategies for weight loss and prevention of weight regain for adults. Med Sci Sports Exerc. 2009;41(2):459-71.

15. ColeTJ, Bellizzi MC, Flegal KM, Dietz WH. Establishing a standard definition for child overweight and obesity worldwide: international survey. Br Med J. 2000;320(7244):1240-3.

16. III Diretrizes Brasileiras sobre Dislipidemias e Diretriz de Prevenção da Aterosclerose do Departamento de Aterosclerose da Sociedade Brasileira de Cardiologia. Arq Bras Cardiol. 2001;77(35):1-48.

17. Watts K, Beye P, Siafarikas A, Davis EA, Jones TW, O'Driscoll G, et al. Exercise training normalizes vascular dysfunction and improves central adiposity in obese adolescents. J Am Coll Cardiol. 2004;43(10):1823-7.

18. Davis JN, Tung A, Chak SS, Ventura EE, Byrd-Williams CE, Alexander $\mathrm{KE}$, et al. Aerobic and strength training reduces adiposity in overweight Latina adolescents. Med Sci Sports Exerc. 2009;41(7):1494-503.

19. Lazzer S, BoirieY, Montaurier C, Vernet J, Meyer M, Vermorel M. A weight reduction program preserves fat-free mass but not metabolic rate in obese adolescents. Obes Res. 2004;12(2):233-40.

20. LeMura LM, Maziekas MT. Factors that alter body fat, body mass, and fat-free mass in pediatric obesity. Med Sci Sports Exerc. 2002;34(3):487-96.

21. Wong PC, Chia MY, Tsou IY, Wansaicheong GK, Tan B, Wang $\mathrm{JC}$, et al. Effects of a 12-week exercise training programme on aerobic fitness, body composition, blood lipids and C-reactive protein in adolescents with obesity. Ann Acad Med Singapore. 2008;37(4):286-93.

22. Castilho SD, Cocetti M, Barros Filho AA. Body mass index and body composition in relation to sexual maturation. J Pediatr Endocrinol Metab. 2008;21:127-33. 
23. Fernandez AC, Mello MT, Tufik S, Castro PM, Fisberg M. Influência do treinamento aeróbio e anaeróbio na massa de gordura corporal de adolescentes obesos. Rev Bras Med Esporte. 2004;10(3)152:64.

24. Daniels SR, Morrison JA, Sprecher DL, Khoury P, Kimball TR. Association of body fat distribution and cardiovascular risk factors in children and adolescents. J Circulation. 1999;99(4):541-5.

25. van Greevenbroek MM, Schalkwijk CG, Stehouwer CD. Obesityassociated low-grade inflammation in type 2 diabetes mellitus: causes and consequences. Neth J Med. 2013;71(4):174-87.

26. Ebbert JO, Jensen MD. Fat depots, free fatty acids, and dyslipidemia. Nutrients. 2013;5(2):498-508.

27. Oliveira CL, Mello MT, Cintra IP, Fisberg M. Obesidade e síndrome metabólica na infância e adolescência. Rev Nutr. 2004;17(2):237-45.
28. Zimmermann R, Lass A, Haemmerle G, Zechner R. Fate of fat: the role of adipose triglyceride lipase in lipolysis. Biochim Biophys Acta. 2009;1791(6):494-500.

29. Foschini D, Araújo RC, Bacurau RF, De Piano A, De Almeida SS, Carnier J, et al. Treatment of obese adolescents: the influence of periodization models and ACE genotype. Obesity (Silver Spring). 2010;18(4):766-72.

30. Kraus WE, Slentz CA. Exercise training, lipid regulation, and insulin action: a tangled web of cause and effect. Obesity (Silver Spring). 2009;17 Suppl 3:S21-6.

31. Antunes BMM, Monteiro PA, Silveira LS, Cayres SU, Silva BC, Freitas Jr IF. Effect of concurrent training on risk factors and hepatic steatosis in obese adolescents. Rev Paul Pediatr. 2013;31(3):371-6.

32. BMI graph page. Available at: <https://www.bcm.edu/cnrc-apps/ bodycomp/bmiz2.html>. Access in: March 1st, 2015. 